\title{
Hypomethylation of CD30 CpG islands with aberrant JunB expression drives CD30 induction in Hodgkin lymphoma and anaplastic large cell lymphoma
}

\author{
Mariko Watanabe ${ }^{1, \star}$, Yuji Ogawa ${ }^{1, \star}$, Kinji Itoh ${ }^{2}$, Tukasa Koiwa $^{3}$, Marshall E Kadin $^{4}$, Toshiki Watanabe ${ }^{3}$, \\ Isao Okayasu ${ }^{5}$, Masaaki Higashihara ${ }^{1}$ and Ryouichi Horie ${ }^{1}$
}

High expression of CD30 and JunB is the hallmark of malignant cells in Hodgkin lymphoma $(\mathrm{HL})$ and anaplastic large cell lymphoma ( $A L C L)$. Ligand-independent signaling by $C D 30$ induces JunB, which activates the CD30 promoter, stabilizing CD30 expression and supporting the survival of Hodgkin-Reed-Sternberg (H-RS) and ALCL cells. Here we show for the first time CpG islands encompassing $60 \mathrm{CpG}$ dinucleotides, located in the core promoter, exon 1 and intron 1 of CD30 gene. Analysis of the methylation status of CD30 CpG islands in H-RS, ALCL and unrelated cell lines reveals an inverse relationship between the extent of CD30 CpG methylation and CD30 expression. CD30 CpG islands of $\mathrm{H}-\mathrm{RS}$ and $\mathrm{ALCL}$ cell lines are rarely methylated. Methylation of the CD30 promoter decreases CD30 induction and JunB action on the demethylated CD30 promoter enhances CD30 induction. CD30 and JunB are strongly expressed in H-RS and ALCL cells, whereas they are not expressed in nonmalignant lymphocytes in which CD30 CpG islands are rarely methylated. We conclude that constitutive action of aberrantly expressed JunB on hypomethylated CD30 CpG islands of lymphocytes triggers CD30 induction and initiates activation of the JunB-CD30-JunB loop, essential to the pathogenesis of $\mathrm{HL}$ and ALCL.

Laboratory Investigation (2008) 88, 48-57; doi:10.1038/labinvest.3700696; published online 29 October 2007

KEYWORDS: CD30; CpG methylation; JunB; Hodgkin lymphoma; anaplastic large cell lymphoma

CD30 is a member of the tumor necrosis factor (TNF) receptor superfamily initially identified on the surface of Hodgkin and Reed-Sternberg (H-RS) cells of Hodgkin lymphoma (HL). ${ }^{1-3}$ Immunohistochemical analysis of a large range of human tumors has shown that CD30 is overexpressed not only by H-RS cells, but also by a subset of diffuse large cell neoplasms with anaplastic features called anaplastic large cell lymphoma (ALCL). ${ }^{4,5}$

Overexpression of CD30 is a characteristic of H-RS and ALCL cells. Ligand-independent signals triggered by overexpressed CD30 induce activation of NF- $\kappa \mathrm{B}$ and the extracellular signal-regulated kinase (ERK) 1/2 mitogen-activated protein kinase (MAPK) pathway, both of which contribute to tumorigenesis and maintenance of survival of H-RS and ALCL cells. ${ }^{6-9}$
Approximately $98 \%$ of $\mathrm{H}-\mathrm{RS}$ cells are derived from B cells and the remaining $2 \%$ of $\mathrm{H}-\mathrm{RS}$ cells appear to be derived from $\mathrm{T}$ cells. ${ }^{10,11}$ Amplification of genomic DNA and complementary DNA (cDNA) derived from single H-RS cells indicates a germinal center B-cell origin of most $\mathrm{H}-\mathrm{RS}$ cells. However, the origin of H-RS cells with T-cell phenotype and that of ALCL cells is entirely unknown. Despite lack of information about the cell origin in T-cell HL and ALCL with CD30 overexpression, it is assumed H-RS and ALCL cells are derived from normal lymphocytes, which lack CD30 expression at resting state. Consequently, understanding the mechanisms for constitutive overexpression of CD30 is important to understand the pathogenesis of HL and ALCL.

Cloning and characterization of the promoter region of the CD30 gene enabled us to demonstrate that the CD30

\footnotetext{
'Department of Hematology, School of Medicine, Kitasato University, Sagamihara, Kanagawa, Japan; ${ }^{2}$ Department of Pathology, School of Medicine, Toho University, Ohta-ku, Tokyo, Japan; ${ }^{3}$ Laboratory of Tumor Cell Biology, Department of Medical Genome Sciences, Graduate School of Frontier Sciences, The University of Tokyo, Minato-ku, Tokyo, Japan; ${ }^{4}$ Department of Dermatology and Skin Surgery, Roger Williams Medical Center, Providence, RI, USA and ${ }^{5}$ Department of Pathology, School of Medicine, Kitasato University, Sagamihara, Kanagawa, Japan

Correspondence: Dr R Horie, MD, PhD, Department of Hematology, School of Medicine, Kitasato University, 1-15-1 Kitasato, Sagamihara, Kanagawa 228-8555, Japan. E-mail: rhorie@med.kitasato-u.ac.jp

*These authors contributed equally to this work.

Received 15 September 2007; accepted 12 October 2007
} 
promoter is composed of a microsatellite sequence (MS) containing CCAT repeats and a core promoter with Sp-1binding sites. The Sp- 1 site at -45 to -39 within core promoter is responsible for basal promoter activity. ${ }^{12,13}$ On the other hand, the CCAT motif repeated in the CD30 MS represses the core promoter activity of $\mathrm{CD} 30$. We found that action of JunB on the AP-1 site in the upstream region of the $\mathrm{CD} 30$ core promoter is responsible for strong activity of the CD30 promoter in H-RS and ALCL cells. ${ }^{14}$ We also showed ligand-independent CD30-ERK-MAPK signals induce amplification of JunB, which acts on the CD30 promoter to stabilize overexpression of $\mathrm{CD} 30$ in $\mathrm{H}-\mathrm{RS}$ and ALCL cells. ${ }^{9}$

Methylation of dinucleotide cytosine-guanosine motifs $(\mathrm{CpG})$, especially in $\mathrm{CpG}$ islands located within promoter regions, is a common mechanism of gene regulation. Until now, there was no knowledge of the role of epigenetic regulation of CD30 gene expression. To learn about the role of epigenetic regulation in CD30 overexpression, we analyzed the CD30 gene and identified CpG islands encompassing $60 \mathrm{CpG}$ dinucleotides (CD30 CpG islands) within the core promoter and exon 1 and intron 1 of the $\mathrm{CD} 30$ gene. We studied the contribution of the methylation status of CD30 CpG islands and JunB expression levels to CD30 overexpression in CD30-positive and -negative cell lines including H-RS cells, ALCL cells, as well as in normal lymphocytes and germinal center cells. We confirmed our findings in tissue sections of HL and ALCL and nonmalignant lymphoid tissues.

\section{MATERIALS AND METHODS Cell Cultures}

K562, HEK293, Jurkat, HeLa, ML1, ML2, FL and A549 cell lines were obtained from the Japanese Cancer Research Resources Bank (Tokyo, Japan) and Fujisaki Cell Biology Center (Okayama, Japan). ALCL cell lines (SUDHL1 and Karpas299) and H-RS cell lines (L428, KMH2, HDLM2, L540 and HDMYZ) were purchased from the German Collection of Microorganisms and Cell Cultures (Braunschweig, Germany). Nonadherent cell lines were cultured in RPMI 1640 and adherent cells in Dulbecco's modified Eagle's medium (DMEM) with supplementation of recommended concentrations of fetal calf serum (FCS) and antibiotics. Normal lymphocytes were separated from peripheral blood of healthy volunteers by differential centrifugation through Lymphoprep (AXIS SHIELD PoC AS, Oslo, Norway). Cells were washed with phosphate-buffered saline $(\operatorname{PBS}(-))$ before use.

\section{Northern Blotting}

Northern blot analysis was carried out essentially as described. ${ }^{15}$ Briefly, $1 \mu \mathrm{g}$ per lane of poly (A)-selected RNA was size-fractionated by $1 \%$ formalin agarose gel electrophoresis and subsequently blotted onto Hybond-C extra nitrocellulose membranes (Amersham Bioscience,
Piscataway, NJ, USA). Filters were hybridized in $4 \times$ SSC, $1 \times$ Denhardts, $0.5 \%$ sodium dodecyl sulfate (SDS), $0.1 \mathrm{M}$ $\mathrm{NaPO} 4(\mathrm{pH} \quad 7.0), 10 \%$ Dextran $\mathrm{Na}$ at $65^{\circ} \mathrm{C}$ with $1.0 \times 10^{6} \mathrm{cpm} / \mathrm{ml}$ of random prime-labeled probes. After washing to a final stringency of $0.2 \times$ SSC and $0.1 \%$ SDS at $65^{\circ} \mathrm{C}$, filters were exposed to XAR-5 films (Eastman Kodak, Rochester, NY, USA) at $-80^{\circ} \mathrm{C}$. RT-PCR-amplified fragments of human CD30 and human GAPDH were used as probes.

\section{Immunohistochemistry}

Cultured cells were immunostained with antibodies for CD30 and JunB, and fluorescence signals were detected using confocal microscopy. Cells were first washed three times with PSB (-). Cytospin samples were prepared using $5 \times 10^{4}$ cells and fixed with $100 \%$ methanol for $10 \mathrm{~min}$ at room temperature, then cells were washed three times in PBS (-). Samples were incubated with primary antibody at the concentration of $5 \mu \mathrm{g} / \mathrm{ml}$ at $4^{\circ} \mathrm{C}$ for overnight and washed with PBS (-) three times. After incubation with fluorescencelabeled secondary antibody for $30 \mathrm{~min}$ at $37^{\circ} \mathrm{C}$, samples were washed three times in PBS (-) and covered with a Perma Fluloro antifade reagent (Therme Shandon. Co., Pittsburgh, PA, USA). Fluorescence signals were detected using confocal microscopy (Radience, 2000) (Bio-Rad Laboratories, Hercules, CA, USA). Antibodies used were as follows; anti-CD30 mouse monoclonal antibody (Ber-H2) (DAKO Kyoto, Japan) and anti-JunB mouse monoclonal antibody (C-11) (Sata Cruz Biotechnology, Inc., Santa Cruz, CA, USA).

Immunostaining of JunB and CD30 was performed on paraffin-embedded specimens of normal peripheral blood mononuclear cell (PBMC) and tonsil as well as lymph nodes affected with HL or ALCL. Prior to incubation of the antiJunB antibody (C-11) or anti-CD30 antibody (Ber-H2), a heat-induced antigen retrieval was performed. Immunodetection was carried out with biotinylated goat antimouse IgG, followed by peroxidase-labeled streptavidine (DAKO). HISTOFINE ${ }^{\mathrm{TM}}$ kit (Nichirei, Tokyo, Japan) was used to detect the color reaction for peroxidase, according to the manufacturer's instructions.

\section{Immunoblotting}

Immunoblot analysis was carried out as described. ${ }^{9}$ Antibodies used were as follows; anti-CD30 antibody (Ber-H2) (DAKO) and anti- $\alpha$ tubulin mouse monoclonal antibody (TU-02) (Santa Cruz). Alkaline phosphatase-conjugated secondary antibodies are as follows; anti-mouse IgG (H\&L) antibody (Promega Madison, WI, USA) and anti-mouse IgM antibody (Santa Cruz).

\section{CpG Methylation Analysis of Cell Lines}

Methylation of the cytosine residue of the CpG site was analyzed by the bisulfite genomic sequencing method with slight modifications. ${ }^{16}$ Briefly, genomic DNA of cell culture samples was extracted by SDS/proteinase $\mathrm{K}$ digestion, 
followed by standard phenol-chloroform extraction and ethanol precipitation. Five $\mu \mathrm{g}$ of genomic DNA was used for bisulfite treatment. The DNA sample in $0.3 \mathrm{~N} \mathrm{NaOH}$ was heat denatured at $75^{\circ} \mathrm{C}$ for $20 \mathrm{~min}$, followed by incubation at $55^{\circ} \mathrm{C}$ for $4 \mathrm{~h}$ in $4.8 \mathrm{M} \mathrm{Na}_{2} \mathrm{~S}_{2} \mathrm{O}_{5}$ and $0.5 \mathrm{mM}$ hydroquinone (both from Sigma, St Louis, MO, USA). The sample DNA was purified using the Wizard DNA Clean-Up system (Promega, Madison, WI, USA) and treated with $0.3 \mathrm{~N} \mathrm{NaOH}$ at $37^{\circ} \mathrm{C}$ for $20 \mathrm{~min}$. DNA was precipitated with ethanol and dissolved in $50 \mu \mathrm{l}$ of $\mathrm{H}_{2} \mathrm{O}$, and $1 / 20$ of this solution was subjected to PCR amplification using the AccuPrime Taq DNA Polymerase System (Invitrogen Graningen, Netherlands). PCR was conducted with a set of primers indicated in Figure 1c as follows: forward primer, 5'-TAAGGGTATGG GAGAAGGTTT- $3^{\prime}$; reverse primer, 5'-CCTCCCACCTA TAAATACTAAC- $3^{\prime}$ (converted cytosine residues are written in bold letters). All primer sequences were devoid of $\mathrm{CpG}$ dinucleotides to avoid biased amplification of methylated alleles. The reaction condition was as follows: initial denature at $94^{\circ} \mathrm{C}$ for $2 \mathrm{~min}, 45 \mathrm{cycles}$ of $94^{\circ} \mathrm{C}$ for $30 \mathrm{~s}, 55^{\circ} \mathrm{C}$ for $30 \mathrm{~s}$, $68^{\circ} \mathrm{C}$ for $1 \mathrm{~min}$ and the final extension at $68^{\circ} \mathrm{C}$ for $7 \mathrm{~min}$. Amplified and gel-purified PCR products were cloned into pGEM-T Easy vector (Promega), and nucleotide sequences of 10 or more clones were determined.

\section{Bisulfite DNA Sequencing Analysis}

For DNA sequencing, we used an ABI PRISM 377 DNA Sequencer (Applied Biosystems, Foster City, CA, USA) and DYEnamic ET Terminator Cycle Sequencing Kit (Amersham Bioscience, Piscataway, NJ, USA).

\section{5-Azacytidine Treatment}

For 5-azacytidine (5-AzaC) (Sigma) treatment, HDMYZ, Jurkat, ML1, FL and HEK293 were seeded at a density of $10^{5}$ cells $/ \mathrm{ml}$, and were treated with the demethylating agent 5AzaC at 1 or $2 \mu \mathrm{M}$ for $72 \mathrm{~h}$ at $37^{\circ} \mathrm{C}$ in a $5 \% \mathrm{CO}_{2}$ humidified atmosphere. Then, 5-AzaC was removed and cells were cultured for $48 \mathrm{~h}$.

\section{Reverse Transcriptase-PCR Analysis}

Total RNA from culture cells was extracted using the QIAzol Lysis Reagent (QIAGEN GmbH, Hilden, Germamy) according to the manufacturer's instruction. Each reaction mixture contained $2 \mu \mathrm{g}$ total RNA, $50 \mathrm{pmol}$ oligo (dT) primer and $200 \mathrm{U}$ SuperScript II reverse transcriptase (Invitrogen) in a $20 \mu \mathrm{l}$ cocktail. After denaturation of RNA at $70^{\circ} \mathrm{C}$ for $10 \mathrm{~min}$, reaction mixtures were pre-incubated for $2 \mathrm{~min}$ at $42^{\circ} \mathrm{C}$, then incubated at $42^{\circ} \mathrm{C}$ with reverse transcriptase for $50 \mathrm{~min}$ and finally denatured at $70^{\circ} \mathrm{C}$ for $15 \mathrm{~min}$. PCR amplifications were performed using Takara Ex Taq DNA Polymerase System (Takara, Kyoto, Japan) according to the manufacturer's instruction. Each specific primers used were follows: CD30 forward, 5'-CAGCTGAGGAGTGGTGCGTCGG-3';

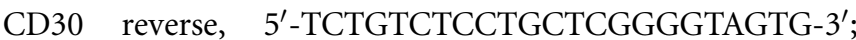
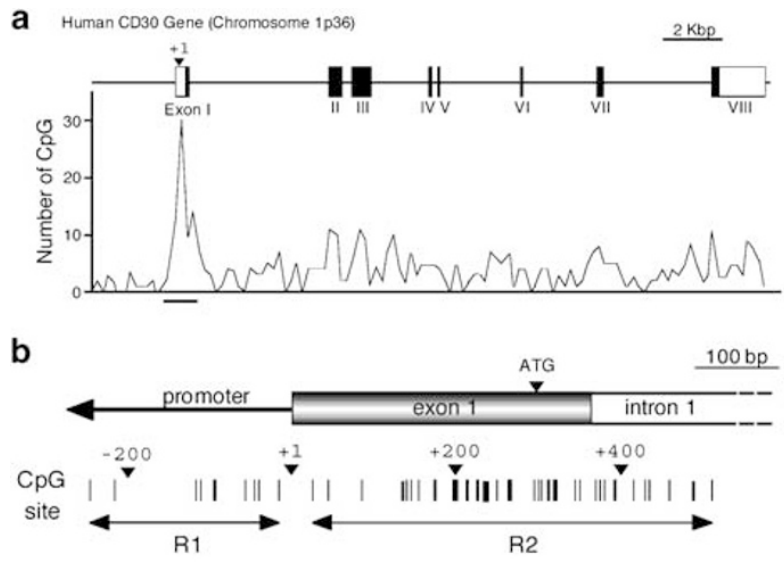

C

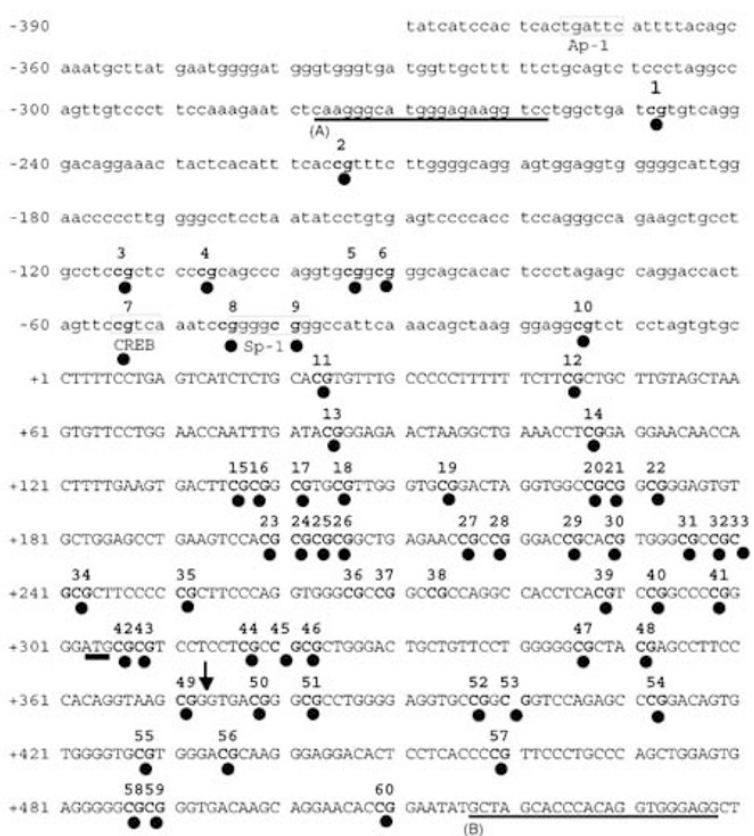

Figure $1 \mathrm{Map}$ of $\mathrm{CpG}$ methylation sites in the promoter, exon 1 and intron 1 of the CD30; primer design for bisulfite sequencing. (a) The line graph indicates the number of $\mathrm{CpG}$ sites per 200 nucleotides in the $\mathrm{CD} 30$ gene. The region analyzed is indicated by a horizontal line. (b) Individual CpG sites analyzed are indicated as vertical lines and numbered from 1 to 60 . Two clusters of $\mathrm{CpG}$ sites are indicated as R1 and R2. (c) Sequence of the $\mathrm{CD} 30$ genomic region analyzed. $\mathrm{CpG}$ sites are indicated by closed circles and numbered. The $5^{\prime}$ transcription start site is designated as +1 and initiation codon (ATG) at +303 is underlined. The primers for PCR are underlined ( $\mathbf{a}$ and $\mathbf{b}$ ). The border between exon 1 and intron 1 is indicated by an arrow. Guanine at the nucleotide sequence +168 was reported as cytosine in the original report. ${ }^{31}$ Potential transcription factor-binding sites are indicated.

$\beta$ actin forward, $5^{\prime}$-TCAGAAGGACTCCTATGTGG-3'; $\beta$ actin reverse, 5'-TCTCTTTGATGTCACGCACG-3'.

\section{In Vitro Methylation and Reporter Gene Assays}

Activities of the CD30 promoter were studied by transient reporter gene assays. CD30 genomic region $(-272 \sim+531)$ or $(-390 \sim+531)$ was amplified by PCR with Takara LA Taq 
polymerase using genomic DNA extracted from KMH2 cells and introduced into an MluI/HindIII site of pGL3Basic vector (Promega). Constructs were methylated in vitro with CpG Methylase (S.ssI) as recommended by the manufacture (New England Biolabs, Ipswich, MA, USA). The methylation status was checked by digestion with restriction enzymes HapII and HhaI. Renilla luciferase expression vector driven by the herpes simplex virus thymidine kinase promoter, pRLTK (Promega), was co-transfected to standardize transfection efficiency in each experiment. Luciferase activities were measured by Dual Luciferase assay kit (Promega). Transfection was carried out using Lipofectamine 2000 Reagent (Invitrogen) according to the manufacture's instructions.

\section{Laser Capture Microdissection, DNA Extraction and Bisulfite Treatment of Clinical Samples}

Formalin-fixed, paraffin-embedded sections were prepared from diagnostic biopsy samples of patients after informed consent. H-RS cells and ALCL cells, which were identified by staining with anti-CD30 antibody Ber-H2 (DAKO) and hematoxylin were microdissected using consecutive sections with modest staining by hematoxylin alone. Briefly, sections were dipped in xylene for $20 \mathrm{~min}$, three changes of xylene for $20 \mathrm{~s}$, three changes of $100 \%$ ethanol for $20 \mathrm{~s}$, dipped in distilled water, three changes of $100 \%$ ethanol for $30 \mathrm{~s}$ and three changes of xylene for $20 \mathrm{~s}$ each. The sections were stained with hematoxylin lightly and after air-drying for $60 \mathrm{~min}$, cells were microdissected using ARCTURUS LM200 Laser Capture Microdissection (LCM) unit (ARCTURUS, Mountain View, CA, USA) or Leica AS LMD microsystem (Leica Microsystems, Tokyo, Japan). DNA was extracted from microdissected samples after digestion with the PicoPure DNA Extraction Kit (ARCTURUS) followed by extraction with phenol-chloroform and precipitation with ethanol. Next, bisulfite-modified analysis was performed by MethylEasy DNA Bisulfite Modification Kit (Human Genetic Signatures Pty Ltd, Australia).

\section{Statistical Analysis}

Differences between mean values were assessed by two-tailed $t$-test. A $P$-value $<0.05$ was considered to be statistically significant.

\section{Gene Bank Accession Number}

The National Center for Biotechnology Information human genome sequence for CD30 is AJ 272029.

\section{RESULTS}

Identification of CpG Islands within the Core Promoter, First Exon and Intron of the CD30 Gene

Methylation of CpG islands located within the promoter region is one mechanism of epigenetic gene regulation. In order to address whether CD30 gene can be regulated epigenetically by $\mathrm{CpG}$ methylation, we analyzed the $\mathrm{CpG}$ sites in the $5^{\prime}$-region of CD30 gene. The analysis revealed
CpG-rich sequences encompassing $60 \mathrm{CpG}$ dinucleotides expanding a region within the promoter, the first exon and the first intron of the CD30 gene (Figure 1a). We identified two CpG islands (island R1, -249 to -15 ; island R2, +23 to +509 ). We deduced that CpG dinucleotides, 1-10 (from $-249 \sim-15)$ are located within the core promoter region and $\mathrm{CpG}$ dinucleotides, 11-60 are located within the first exon and a part of the first intron of CD30 gene (from $+23 \sim+509$ ) (Figure 1b). Primers designed for PCR to amplify the 816 bp sequence that encompasses these $60 \mathrm{CpG}$ sites were underlined ( $A$ and $B$ in Figure 1c). Bisulfite sequencing was used to study the methylation status of each $\mathrm{CpG}$ site within this region.

\section{Expression of CD30 mRNA and Proteins in H-RS, ALCL and Unrelated Cell Lines}

We first examined the expression of CD30 mRNA and protein in H-RS cell lines (L428, KMH2, HDLM2, L540 and HDMYZ), ALCL cell lines (SUDHL1 and Karpas299) and unrelated cell lines used in this study. Unrelated cell lines examined include Jurkat (T-cell lymphoblastic lymphoma/ leukemia), K562, ML1 and ML2 (all myeloid leukemia), HEK293 (embryonic kidney), FL (embryonal epithelium), HeLa (uterine cervical carcinoma) and A549 (lung adenocarcinoma). Among cell lines tested, all H-RS cell lines, except for HDMYZ, and all ALCL cell lines showed strong CD30 mRNA and protein expression. Among unrelated cell lines, Jurkat and K562 expressed lower levels of CD30 than H-RS or ALCL cells. Cell lines HeLa, ML1, ML2, HEK293, FL and A549 did not express CD30 (Figure 2a). These observations are in agreement with previous observations that CD30 is strongly expressed in H-RS and ALCL cells and absent or expressed at low levels in some hematopoietic cells, and not expressed in most nonhematopoietic cells. ${ }^{5}$ Although HDMYZ is reported as a H-RS cell line, this cell line lacks several characteristics of $\mathrm{H}-\mathrm{RS}$ cells including lack of CD30 expression.

\section{Methylation Status of CD30 CpG Islands in H-RS, ALCL and Unrelated Cell Lines}

In order to map the methylation sites within the CD30 CpG islands, we treated DNA from H-RS cell lines, ALCL cell lines and unrelated cell lines with bisulfite, which chemically converts unmethylated cytosine to uracil, whereas it has no effect on methylated cytosine, that is in CpG. PCR amplification, cloning and sequencing of bisulfite DNA showed a specific methylation pattern of the analyzed $60 \mathrm{CpG}$ sites in all cell lines (Figure $2 \mathrm{~b}$ and Table 1). In general, methylation status could be classified into cell lines with rarely methylated CD30 CpG islands (L428, KMH2, L540, HDLM2, Karpas299 and SUDHL1), those with frequently methylated CD30 CpG islands (HeLa ML1, ML2, HEK293, FL and A549) and those with partially methylated CD30 CpG islands (HDMYZ, Jurkat and K562). Interestingly, all cell lines with rarely methylated CD30 CpG islands were derived from HL or 
a

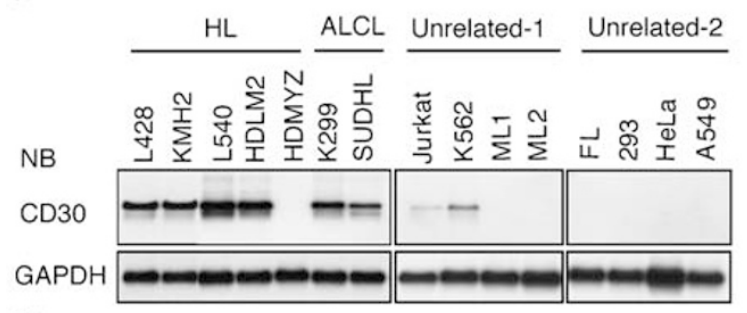

IB

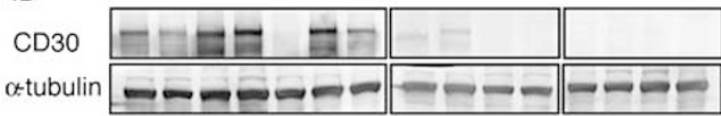

b

HL

L428

KMH2

L540

HDLM2

HDMYZ

ALCL

Karpas299
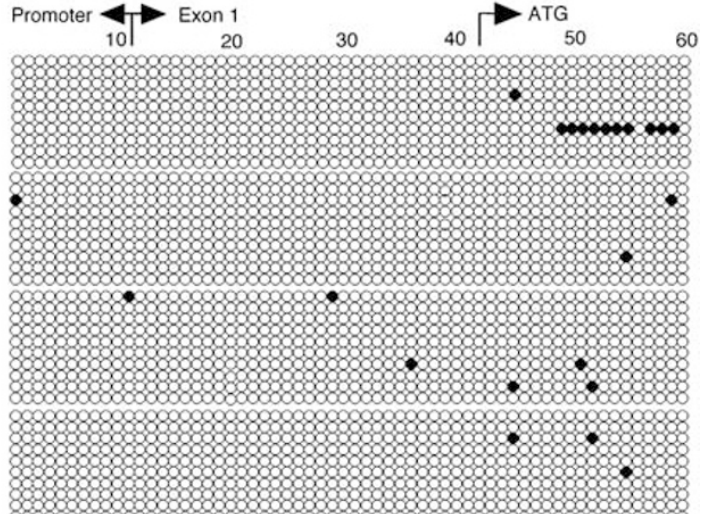

gewe 8
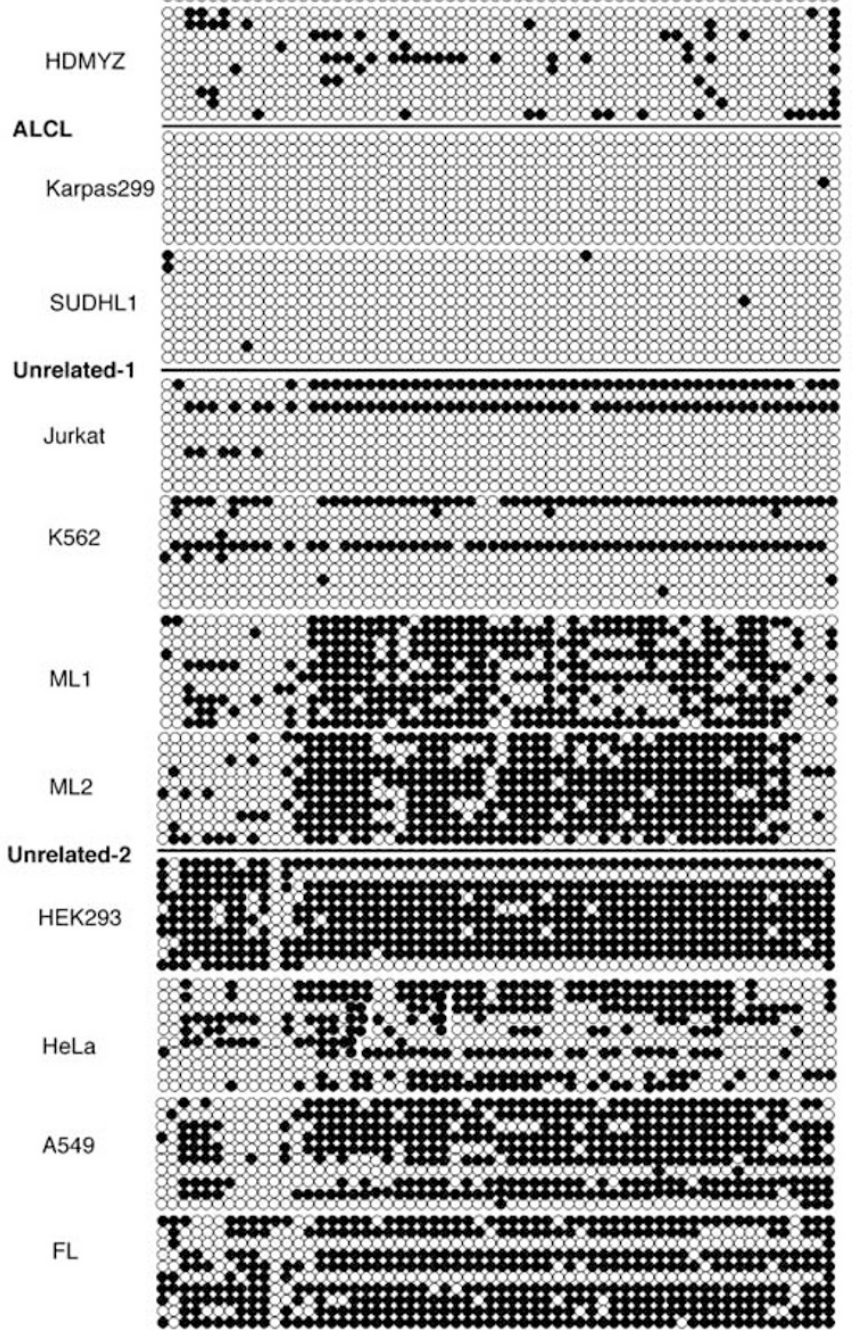

Figure 2 The expression level of CD30 and the status of CD30 CpG island methylation in H-RS, ALCL and unrelated cell lines. (a) Northern blot analysis of CD30. One microgram of polyadenylic acid-selected RNA was subjected to analysis. Glyceraldehyde-3-phosphate dehydrogenase (GAPDH) served as a control (top). Immunoblot analysis of CD30. Twenty micrograms of whole-cell lysates were subjected to analysis. Alpha tubulin served as a control (bottom). Cell lines used are indicated above the lanes. NB, northern blot analysis; IB, immunoblot analysis. (b) Results of bisulfite genomic sequencing coupled with TA cloning are shown. The methylation status of 10 clones for each sample is presented. Each circle represents a CpG site, a filled circle indicates methylation and an open circle indicates no methylation. Cell lines used are indicated on the left. The numbers described above correspond to those indicated in Figure 1.

ALCL, which are strongly CD30 positive. The percentage of CpG methylation in CD30 CpG islands from CD30-positive cells was very low (mean 5.3\%) as compared with CD30- negative cells (mean 55.1\%) and statistically significant $(P<0.01)$ (Table 1). These results indicate involvement of $\mathrm{CpG}$ methylation in the regulation of CD30 expression. 
Table 1 Methylation pattern of CD30 CpG islands

\begin{tabular}{|c|c|c|c|c|}
\hline \multirow[t]{2}{*}{ Cell lines } & \multirow[t]{2}{*}{ CD30 } & \multicolumn{3}{|c|}{ Methylated CpG (\%) } \\
\hline & & Total & $\mathrm{R} 1$ & $\mathrm{R} 2$ \\
\hline L428 & + & 1.8 & 0.0 & 2.2 \\
\hline $\mathrm{KMH} 2$ & + & 0.5 & 1.0 & 0.4 \\
\hline L540 & + & 1.0 & 0.0 & 1.2 \\
\hline HDLM2 & + & 0.5 & 0.0 & 0.6 \\
\hline Karpas299 & + & 0.2 & 0.0 & 0.2 \\
\hline SUDHL1 & + & 0.8 & 3.0 & 0.4 \\
\hline Jurkat & + & 17.7 & 12.0 & 18.8 \\
\hline K562 & + & 19.7 & 23.0 & 19.0 \\
\hline Mean & & 5.3 & 4.9 & 5.3 \\
\hline HDMYZ & - & 11.8 & 13.0 & 11.6 \\
\hline ML1 & - & 58.9 & 21.0 & 66.2 \\
\hline ML2 & - & 66.3 & 17.0 & 76.2 \\
\hline A549 & - & 60.3 & 29.0 & 70.2 \\
\hline HeLa & - & 42.8 & 22.0 & 47.0 \\
\hline HEK293 & - & 76.8 & 85.0 & 66.6 \\
\hline $\mathrm{FL}$ & - & 68.8 & 62.0 & 75.2 \\
\hline Mean & & 55.1 & 35.6 & 59.0 \\
\hline
\end{tabular}

+, positive; - , negative; total, $R 1+R 2$.

Methylated CpG (\%) = (number of CpG sites methylated/total CpG sites) $\times 100$.

The data presented are average of 10 clones analysed. R1 and R2 are indicated in Figure 1.

Among cell lines with frequently methylated CD30 CpG islands, relatively low frequency of methylation of R1 (promoter region) was found in ML1, ML2, HeLa and A549 (mean 22.3\%), whereas methylation of R1 region frequency was high in HEK293 and FL (mean 73.5\%) (Figure 2b and Table 1). Methylaton frequency of $\mathrm{CpG}$ sites within R2 (intron 1 and exon1) is almost equally high in these six cell lines.

\section{Methylation of CD30 CpG Islands Decreases the Expression of CD30}

To further examine whether methylation status of CD30 CpG islands affects the expression of CD30, we treated cell lines with modest CD30 expression (Jurkat) and cell lines without CD30 expression (ML1, HEK293 and FL) by 5-AzaC, which inhibits de novo and maintenance methylation. Treatment with 5-AzaC increased CD30 mRNA expression in cell lines with modest CD30 mRNA expression (Figure 3a). Notably, treatment with 5-AzaC induced CD30 mRNA expression in cell lines (ML1, HEK293 and FL) without constitutive CD30 expression (Figure 3a). Analysis of the methylation status of CD30 CpG islands in Jurkat revealed decreased CpG methylation levels after 5-AzaC treatment (Figure 3b). These a


C

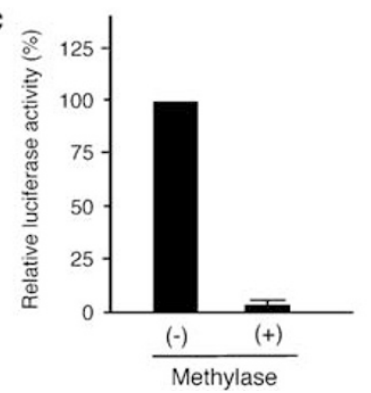

d

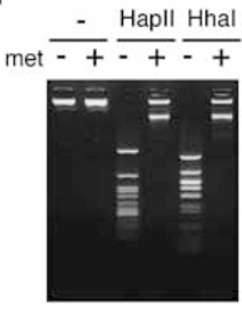

Figure 3 Regulation of CD30 expression by methylation. (a) Induction of CD30 expression after demethylation by 5-AzaC. Cells were treated with 5AzaC at $1 \mu \mathrm{M}$ (Jurkat) or $2 \mu \mathrm{M}$ (ML1, HEK293, and FL) for $72 \mathrm{~h}$ at $37^{\circ} \mathrm{C}$ in a $5 \%$ $\mathrm{CO}_{2}$ humidified atmosphere. Then, $5-\mathrm{AzaC}$ was removed and cells were cultured for $48 \mathrm{~h}$. Using CDNA obtained from these cell lines, the difference in expression level of CD30 mRNA transcripts without $(-)$ or with $(+)$ 5AzaC treatment was examined by RT-PCR. After 35 cycles of amplification, products were analyzed by $3 \%$ agarose gel electrophoresis. (b) Representative methylation patterns of CD30 CpG islands in Jurkat lymphoblastic cells before and after 5-Aza C treatment. (c) Effect of introduction of methylation in transcriptional control of the CD30 promoter. Reporter gene assays were carried out using Jurkat cells and reporter plasmid containing CD30 CpG islands ( -272 to +531 in Figure 1c) treated with or without S.ssI methylase. Relative activity of CD30 promoter treated with S.ssI methylase is shown. Activity of untreated CD30 promoter served as a control, which was set to $100 \%$. (d) Restriction analysis of the methylated reporter construct. The construct before and after methylation was digested with methylation-sensitive endonucleases (Hapll and Hhal).

observations indicate that decreased methylation status triggers expression of CD30. To provide evidence for the direct effect of methylation on CD30 promoter activity, we performed a reporter gene assay with $\mathrm{CD} 30$ promoter constructs (nucleic acid position at $-276 \sim+531$ in Figure 1c) before and after in vitro methylation. Treatment with S.ssI methylase significantly reduced transcriptional activity of the CD30 promoter, suggesting that the introduction of methylation suppresses activity of the CD30 promoter (Figure 3c). Methylation of the construct was confirmed by enzyme restriction assay (Figure 3d). These observations indicate that methylation of CD30 CpG islands reduces the expression of CD30. 

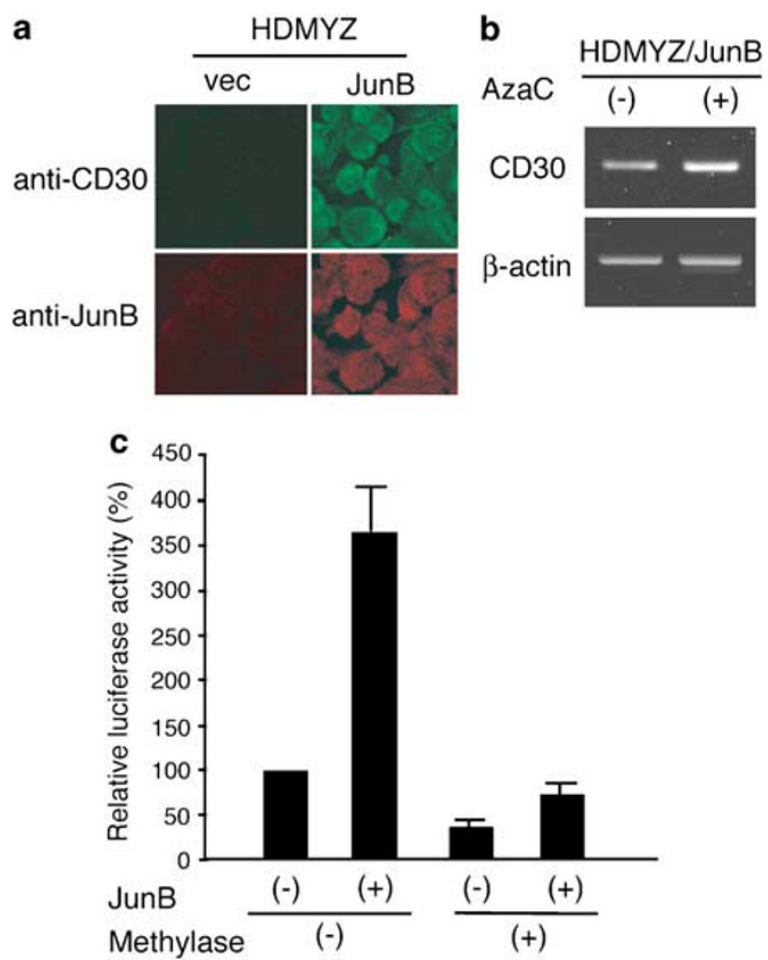

Figure 4 Roles of JunB and methylation status of CD30 GpG islands in CD30 induction. (a) Induction of CD30 in HDMYZ cells with stably transduced JunB. HDMYZ cells $\left(1 \times 10^{7}\right)$ were transfected with $5 \mu \mathrm{g}$ of JunB expression vector or vacant vector using Lipofectoamine 2000 (Invitrogen) and selected by $3 \mu \mathrm{g} / \mathrm{ml}$ of puromycin. Cells cloned were stained with antibodies for CD30 and JunB and analyzed by confocal microscopy. (b) Induction of CD30 expression after 5-AzaC treatment. HDMYZ cells constitutively overexpressing JunB were treated with 5 -AzaC at $1 \mu \mathrm{M}$ for $72 \mathrm{~h}$ at $37^{\circ} \mathrm{C}$ in a $5 \% \mathrm{CO}_{2}$ humidified atmosphere. Then, 5-AzaC was removed and cells were cultured for $48 \mathrm{~h}$. Using CDNA obtained from cells, the difference in expression level of CD30 mRNA transcripts without (-) or with (+) 5-AzaC treatment was examined by RT-PCR. After 35 cycles of amplification, products were analyzed by $3 \%$ agarose gel electrophoresis. $\beta$-Actin served as a control. (c) Effect of introduction of methylation in JunBmediated induction of the CD30 promoter. Reporter gene assays were carried out using HDMYZ cells and $0.2 \mu \mathrm{g}$ of reporter plasmid containing CD30 CpG islands of CD30 gene $(-390 \sim+531$ in Figure $1 \mathrm{c})$ treated with or without S.ssl methylase. In total, $0.5 \mu \mathrm{g}$ of JunB or vacant vector was transfected based on the method described in the Materials and methods. Relative activity of CD30 promoter is shown. Activity of unmethylated CD30 promoter without JunB transfection served as a control, which was set to $100 \%$.

\section{JunB Action on the Demethylated CD30 Promoter Enhances CD30 Induction}

We recently reported that JunB plays an important role in CD30 induction. ${ }^{14}$ Therefore, we examined the effect of CD30 CpG island methylation on JunB-mediated induction of the CD30 promoter. For this purpose we used HDMYZ cells, whose CD30 CpG islands are partially methylated. HDMYZ lacks CD30 expression (Figure 2a) and shows weak JunB expression (data not shown). We produced transformants of HDMYZ cells overexpressing JunB and three independent clones were examined for CD30 expression.
Constitutive overexpression of JunB in HDMYZ cells induced CD30, and 5-Aza C treatment of these clones enhanced CD30 expression. Representative results are shown in Figure 4a and $\mathrm{b}$, respectively. The results indicate that JunB-mediated induction of CD30 is enhanced by reduced methylation status of the CD30 promoter. To confirm the above result, we performed a gene reporter assay with $\mathrm{CD} 30$ promoter constructs (nucleic acid position at $-390 \sim+531$ in Figure 1c) before and after in vitro methylation. JunB-mediated CD30 promoter induction was abolished when the $\mathrm{CD} 30$ promoter treated with S.ssI methylase was used (Figure 4c). The result suggests that constitutive action of overexpressed JunB on reduced methylation of $\mathrm{CD} 30 \mathrm{CpG}$ islands enhances $\mathrm{CD} 30$ promoter induction.

\section{Status of CD30 CpG Island Methylation, JunB and CD30 Expression in H-RS Cells, ALCL Cells and Normal Lymphocytes}

To obtain insight into CD30 induction during transformation of nonmalignant lymphocytes to H-RS and ALCL cells, we examined the methylation status of $\mathrm{CD} 30 \mathrm{CpG}$ islands in H-RS cells, ALCL cells and normal lymphocytes. For this purpose, H-RS cells, ALCL cells and nonmalignant germinal center (GC) cells were microdissected from tissue biopsy samples $(n=3$ for each). PBMC from healthy volunteers were also studied $(n=3)$. We also examined the expression of CD30 and JunB in HL, ALCL, nonmalignant GC and PBMC by immunostaining ( $n=5$ for each). CD30 CpG islands of $\mathrm{H}-\mathrm{RS}$ cells, ALCL cells, GC cells and PBMC were rarely methylated. CD30 and JunB were strongly positive in $\mathrm{H}-\mathrm{RS}$ and ALCL cells, whereas CD30 and JunB were negative in GC cells of tonsils and PBMC. Representative results are shown in Figure $5 \mathrm{a}$ and $\mathrm{b}$. These results indicate that both JunB expression and hypomethylation of $\mathrm{CpG}$ islands are required for CD30 expression.

\section{DISCUSSION}

Methylation of $\mathrm{CpG}$ islands located in gene promoter regions is thought to be one mechanism of gene regulation. For example, aberrant $\mathrm{CpG}$ methylation of the SHP-1 promoter occurs in the pathogenesis of both B- and T-cell lymphomas. ${ }^{17,18}$ Therefore, we investigated the methylation status of the $\mathrm{CD} 30$ promoter, since $\mathrm{CD} 30$ overexpression is essential to the pathogenesis of HL and ALCL. ${ }^{6,919}$ We identified CpG islands encompassing $60 \mathrm{CpG}$ dinucleotides, located in the core promoter, exon 1 and intron 1 of CD30 gene. Our observations in various cell lines reveal that methylation of this region inversely correlates with expression of CD30, suggesting that the $\mathrm{CpG}$ methylation inhibits CD30 expression. This conclusion is supported by the results that treatment with demethylating agent 5-AzaC increases CD30 expression in cell lines with high $\mathrm{CpG}$ methylation, and $\mathrm{CpG}$ methylation of the CD30 promoter by S.ssI methylase decreases transcriptional activity of CD30 promoter. 
a

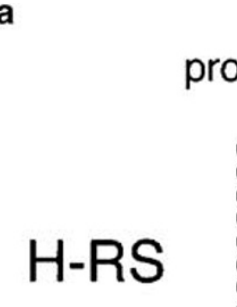



20

30

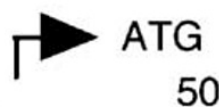

50

60

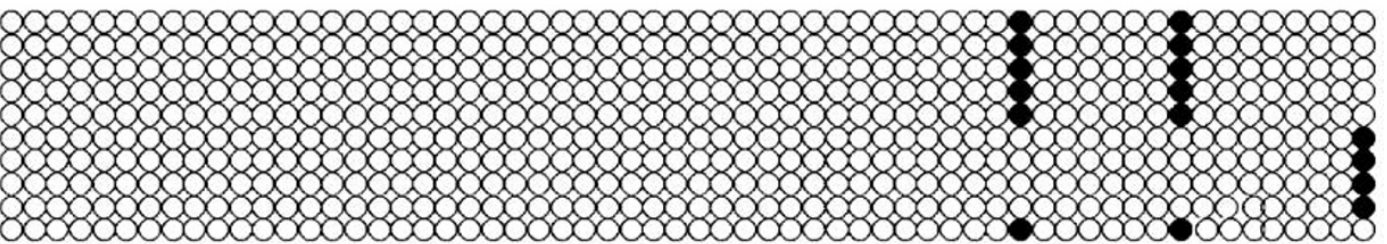

ALCL



$\mathrm{GC}$

PBMC

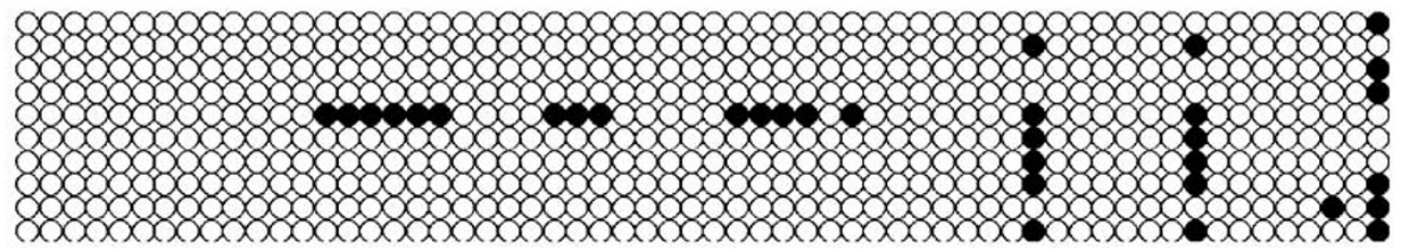

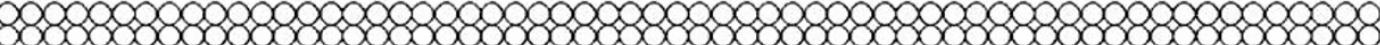
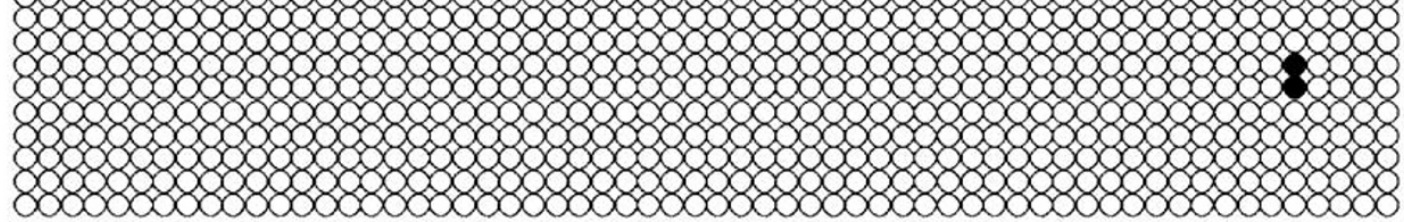

b

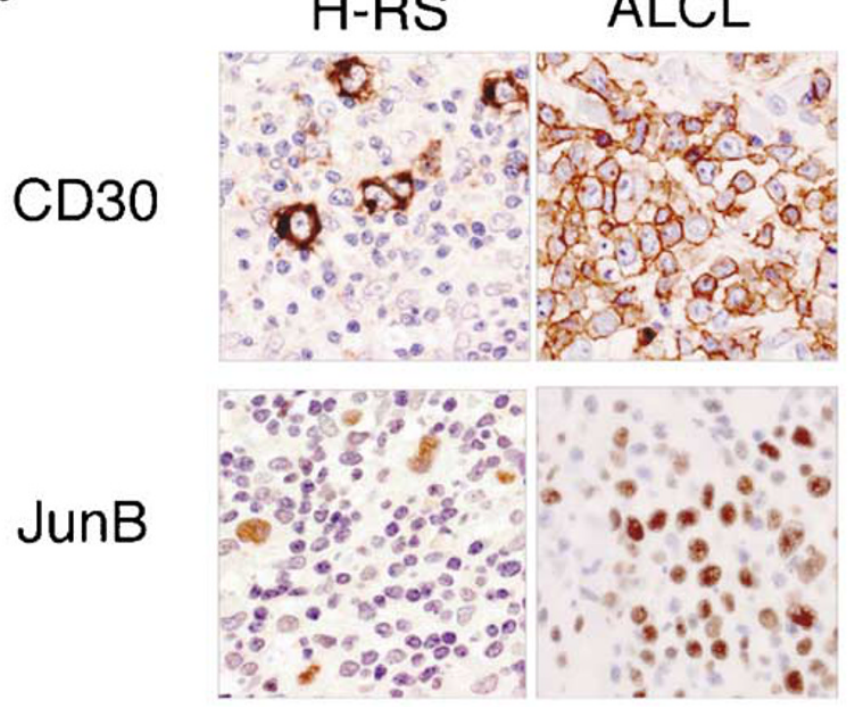

$\mathrm{ALCL}$

(1)
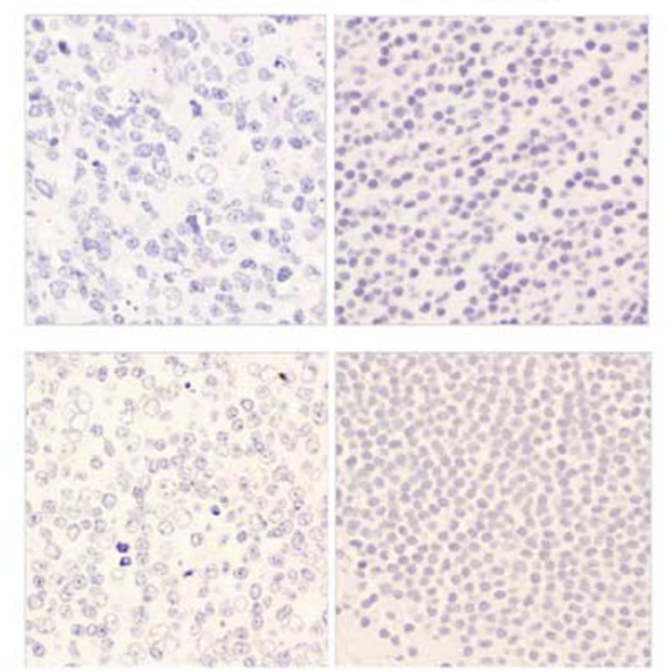

Figure 5 Status of CD30 CpG islands methylation and JunB expression in H-RS cells, ALCL cells and lymphocytes. (a) Analysis of H-RS cells, ALCL cells and GC cells of normal tonsils obtained by microdissection and PBMC by bisulfite genomic sequencing coupled with TA cloning. The methylation status of 10 clones for each sample is represented. Each circle represents a CpG site, a filled circle indicates methylation and an open circle indicates no methylation. Cells used are indicated on the left. The numbers above correspond to those indicated in Figure 1. (b) Expression of JunB and CD30 in $\mathrm{H}-\mathrm{RS}$ cells, ALCL cells, GC cells and PBMC. Tissue samples (HL, ALCL and normal tonsil) and normal PBMC were stained with antibodies for CD30 (top) or JunB (bottom). 
Analysis of the methylation pattern of cell lines with frequently methylated CD30 CpG islands (Figure $2 \mathrm{~b}$ ) indicates that low methylation frequency of $\mathrm{CpG}$ sites within R2 (exon 1 and intron 1) is significant for CD30 expression. On the other hand, the R1 (core promoter) contains GC box and CREB-binding motifs. These motifs in general initiate and promote transcription by recruiting general transcription factors and coactivators. ${ }^{20}$ It has been demonstrated that DNA methylation can interfere with protein-DNA interactions, recruitment of histone deacetylases, and the induction of chromatin condensation necessary for gene inactivation. Several methyl-binding proteins can compete with transcription factors for the same sequences. ${ }^{21,22}$ Therefore, it is likely that the methylation frequency of $\mathrm{CpG}$ sites within $\mathrm{R} 1$ is also critical for CD30 expression. In sum, low methylation frequency of $\mathrm{CpG}$ sites within both R1 and R2 regions appear to be necessary for CD30 induction.

We show that both hypomethylation of CD30 CpG islands and strong JunB expression are required for enhanced CD30 expression. We reported that $\mathrm{CD} 30$ overexpression in $\mathrm{H}-\mathrm{RS}$ cells and ALCL cells is caused by strong AP-1 activity of JunB. Interaction of JunB with the AP-1 site in the $\mathrm{CD} 30$ promoter, which is situated upstream of the R1 region, drives CD30 expression. ${ }^{14}$ However, JunB alone failed to recover activity of the CD30 promoter treated by S.ssI methylase (Figure 4c). JunB overexpression in cell lines with partial methylation could induce CD30 expression which was enhanced by 5-AzaC treatment (Figure 4a and b). Together, these results support the conclusion that both hypomethylation of CD30 CpG islands and JunB overexpression enhance CD30 expression.

Based on the results that the methylation status of CD30 CpG islands critically affect CD30 expression, we hypothesized that CD30 CpG islands in normal germinal center cells and peripheral lymphocytes are methylated and demethylation events directly trigger constitutive CD30 induction. However, our results show that CD30 CpG islands of normal lymphocytes are rarely methylated, suggesting that demethylation of CD30 CpG islands is not sufficient to trigger CD30 induction during lymphomagenesis. Instead, our current results indicate that aberrant induction of JunB at hypomethylated $\mathrm{CD} 30 \mathrm{CpG}$ islands triggers $\mathrm{CD} 30$ induction and initiates an autocrine loop of $\mathrm{CD} 30$-mediated activation of NF- $\kappa \mathrm{B}$ and ERK1/2-MAPK in HL and ALCL. ${ }^{9}$

DNA hypomethylation and mismatch repair deficiency interact to cause invasive T- and B-cell lymphomas in mice. ${ }^{23}$ The exact mechanism for lymphogenesis in these mice is unknown, although enhanced microsatellite instability is likely to be involved. ${ }^{24}$ In the case of HL and ALCL, which are derived from B- and $\mathrm{T}$ lymphocytes, microsatellite instability is uncommon. ${ }^{25,26}$ Instead, we found initiation and constitutive transcription of $\mathrm{CD} 30$ gene by aberrant JunB expression maintains hypomethylation of $\mathrm{CD} 30 \mathrm{CpG}$ islands and contributes to the transformation of lymphocytes to H-RS and ALCL cells. Previous reports support the notion that constitutive transcription of genes inhibits the progression of their methylation. ${ }^{27-30}$

In conclusion, the results of this study indicate that the methylation status of CD30 CpG islands and expression level of JunB together are responsible for high CD30 expression. Combined with our recent findings, these results suggest that constitutive action of JunB on hypomethylated CD30 $\mathrm{CpG}$ islands in lymphocytes triggers CD30 induction and initiates high constitutive activity of a JunB-CD30-JunB loop, maintaining hypomethylation of $\mathrm{CD} 30 \mathrm{CpG}$ islands and contributing to the pathogenesis of HL and ALCL.

\section{ACKNOWLEDGEMENT}

This work was supported by Grants-in-Aid for Scientific Research from Japanese Society for Promotion of Science and a grant from The Mochida Memorial Foundation for Medical and Pharmaceutical Research to Ryouichi Horie.

1. Schwab U, Stein H, Gerdes J, et al. Production of a monoclonal antibody specific for Hodgkin and Sternberg-Reed cells of Hodgkin's disease and a subset of normal lymphoid cells. Nature 1982;299:65-67.

2. Smith CA, Gruss HJ, Davis T, et al. CD30 antigen, a marker for Hodgkin's lymphoma, is a receptor whose ligand defines an emerging family of cytokines with homology to TNF. Cell 1993;73:1349-1360.

3. Durkop $\mathrm{H}$, Latza $\mathrm{U}$, Hummel $\mathrm{M}$, et al. Molecular cloning and expression of a new member of the nerve growth factor receptor family that is characteristic for Hodgkin's disease. Cell 1992;68:421-427.

4. Falini B, Pileri S, Pizzolo G, et al. CD30 (Ki-1) molecule: a new cytokine receptor of the tumor necrosis factor receptor superfamily as a tool for diagnosis and immunotherapy. Blood 1995;85:1-14.

5. Horie R, Watanabe T. CD30: expression and function in health and disease. Semin Immunol 1998;10:457-470.

6. Horie R, Watanabe T, Morishita $Y$, et al. Ligand-independent signaling by overexpressed CD30 drives NF-kappaB activation in HodgkinReed-Sternberg cells. Oncogene 2002;21:2493-2503.

7. Horie $R$, Watanabe $T$, Ito $K$, et al. Cytoplasmic aggregation of TRAF2 and TRAF5 proteins in the Hodgkin-Reed-Sternberg cells. Am J Pathol 2002;160:1647-1654.

8. Nonaka M, Horie R, Itoh K, et al. Aberrant NF-kappaB2/p52 expression in Hodgkin/Reed-Sternberg cells and CD30-transformed rat fibroblasts. Oncogene 2005;24:3976-3986.

9. Watanabe $M$, Sasaki $M$, Itoh $K$, et al. JunB induced by constitutive CD30-extracellular signal-regulated kinase $1 / 2$ mitogen-activated protein kinase signaling activates the CD30 promoter in anaplastic large cell lymphoma and Reed-Sternberg cells of Hodgkin lymphoma. Cancer Res 2005;65:7628-7634

10. Seitz V, Hummel M, Marafioti T, et al. Detection of clonal T-cell receptor gamma-chain gene rearrangements in Reed-Sternberg cells of classic Hodgkin disease. Blood 2000;95:3020-3024.

11. Kanzler $H$, Kuppers $R$, Hansmann ML, et al. Hodgkin and ReedSternberg cells in Hodgkin's disease represent the outgrowth of a dominant tumor clone derived from (crippled) germinal center B cells. J Exp Med 1996;184:1495-1505.

12. Croager EJ, Gout AM, Abraham LJ. Involvement of Sp1 and microsatellite repressor sequences in the transcriptional control of the human CD30 gene. Am J Pathol 2000;156:1723-1731.

13. Croager EJ, Muir TM, Abraham $\amalg$. Analysis of the human and mouse promoter region of the non-Hodgkin's lymphoma-associated CD30 gene. J Interferon Cytokine Res 1998;18:915-920.

14. Watanabe $\mathrm{M}$, Ogawa $\mathrm{Y}$, Ito $\mathrm{K}$, et al. AP-1 mediated relief of repressive activity of the CD30 promoter microsatellite in Hodgkin and ReedSternberg cells. Am J Pathol 2003;163:633-641.

15. Horie $R$, Ito $K$, Tatewaki $M$, et al. A variant $C D 30$ protein lacking extracellular and transmembrane domains is induced in $\mathrm{HL}-60$ by tetradecanoylphorbol acetate and is expressed in alveolar macrophages. Blood 1996;88:2422-2432.

16. Clark SJ, Harrison J, Paul CL, et al. High sensitivity mapping of methylated cytosines. Nucleic Acids Res 1994;22:2990-2997. 
17. Koyama M, Oka $T$, Ouchida $M$, et al. Activated proliferation of B-cell lymphomas/leukemias with the SHP1 gene silencing by aberrant CpG methylation. Lab Invest 2003;83:1849-1858.

18. Zhang $\mathrm{Q}$, Wang HY, Marzec $\mathrm{M}$, et al. STAT3- and DNA methyltransferase 1-mediated epigenetic silencing of SHP-1 tyrosine phosphatase tumor suppressor gene in malignant $\mathrm{T}$ lymphocytes. Proc Natl Acad Sci USA 2005;102:6948-6953.

19. Horie R, Watanabe M, Ishida T, et al. The NPM-ALK oncoprotein abrogates CD30 signaling and constitutive NF-kappaB activation in anaplastic large cell lymphoma. Cancer Cell 2004;5:353-364.

20. Philipsen S, Suske G. A tale of three fingers: the family of mammalian Sp/XKLF transcription factors. Nucleic Acids Res 1999:27:2991-3000.

21. Johnson CA, Turner BM. Histone deacetylases: complex transducers of nuclear signals. Semin Cell Dev Biol 1999;10:179-188.

22. Garinis GA, Patrinos GP, Spanakis NE, et al. DNA hypermethylation: when tumour suppressor genes go silent. Hum Genet 2002;111:115-127.

23. Trinh $\mathrm{BN}$, Long $\mathrm{Tl}$, Nickel $\mathrm{AE}$, et al. DNA methyltransferase deficiency modifies cancer susceptibility in mice lacking DNA mismatch repair. Mol Cell Biol 2002;22:2906-2917.

24. Kim M, Trinh BN, Long $\mathrm{Tl}$, et al. Dnmt1 deficiency leads to enhanced microsatellite instability in mouse embryonic stem cells. Nucleic Acids Res 2004;32:5742-5749.
25. Hodges $\mathrm{KB}$, Vnencak-Jones $\mathrm{CL}$, Larson RS, et al. Rarity of genomic instability in pathogenesis of systemic anaplastic large cell lymphoma (ALCL) in immunocompetent patients. Hum Pathol 1999:30:173-177.

26. Re $D$, Benenson L, Wickenhauser $C$, et al. Proficient mismatch repair protein expression in Hodgkin and Reed Sternberg cells. Int J Cancer 2002;97:205-210.

27. De Smet C, Loriot A, Boon T. Promoter-dependent mechanism leading to selective hypomethylation within the $5^{\prime}$ region of gene MAGE-A1 in tumor cells. Mol Cell Biol 2004;24:4781-4790.

28. Song JZ, Stirzaker C, Harrison J, et al. Hypermethylation trigger of the glutathione-S-transferase gene (GSTP1) in prostate cancer cells. Oncogene 2002;21:1048-1061.

29. Miyamoto K, Fukutomi T, Akashi-Tanaka S, et al. Identification of 20 genes aberrantly methylated in human breast cancers. Int J Cancer 2005;116:407-414.

30. Hagihara A, Miyamoto K, Furuta J, et al. Identification of $275^{\prime} \mathrm{CpG}$ islands aberrantly methylated and 13 genes silenced in human pancreatic cancers. Oncogene 2004;23:8705-8710.

31. Durkop $H$, Oberbarnscheidt $M$, Latza $U$, et al. Structure of the Hodgkin's lymphoma-associated human CD30 gene and the influence of a microsatellite region on its expression in CD30 (+) cell lines. Biochim Biophys Acta 2001;1519:185-191. 